\title{
Amplification of neutrino oscillations by a density ripple in dense plasmas
}

\author{
P. K. SHUK L A \\ RUB International Chair, International Centre for Advanced Studies in Physical Sciences, \\ Institute for Theoretical Physics V, Faculty of Physics \& Astronomy, \\ Ruhr University Bochum, 44780 Bochum, Germany; \\ Scottish Universities Physics Alliance (SUPA), Department of Physics, \\ University of Strathclyde, Glasgow, Scotland; \\ School of Physics, University of KwaZulu-Natal, Durban 4000 Durban, \\ South Africa; and \\ Departamento de Física and Instituto de Plasmas e Fusão Nuclear, Instituto Superior \\ Técnico, Universidade Técnica de Lisboa, 1049-001 Lisboa, Portugal, profshukla@yahoo.de, \\ ps@tp4.rub.de)
}

(Received 19 November 2010 and accepted 26 November 2010, first published online 21 January 2011)

\begin{abstract}
It is shown that a pre-existing electron density ripple in a dense plasma can excite electron neutrino oscillations. For our purposes, we use the dispersion relation for neutrino oscillations and derive the Mathieu equation for the propagation of neutrino oscillations in the presence of a spatially oscillating electron density ripple. The Mathieu equation predicts instability of neutrino oscillations. The criterion under which instability occurs is presented. Analytical expressions for the neutrino oscillation frequency and the growth rate are obtained. The possible relevance of our investigation to non-thermal neutrino oscillations in dense plasma environments (e.g. the supernovae, the core of white dwarf stars etc.) is briefly mentioned.
\end{abstract}

Neutrinos, which are the least massive subatomic ghostly elementary particles in the set of building blocks of nature, were produced in the Big-Bang, which began the universe, and are emitted by the Sun and all other stars (e.g. neutron stars, white dwarf stars, magnetars etc.) These are also produced in violent astrophysical events in cosmos, e.g. explosions of supernovae, which occur when an old massive star collapses after running out of nuclear fuel. During the collapse, the star literally becomes a neutron star as neutrinos totally dominate in numbers of particles for a few seconds and carry off most of the energy from the implosion, more energy than radiated during the entire life of the star. For example, the neutrino flash was actually observed in Supernova 1987A. Neutrinos are also created when cosmic rays, fast moving particles from space, bombard the Earth's atmosphere producing cascades of secondary particles, which rain down on us. There are three different kinds, or 'flavors', as they are called, of neutrinos: electron, muon and tau neutrinos. There are also three anti-neutrinos of the same flavors. The neutrinos get their names from their charged lepton brethren, the electron, muon and tau, with masses of $0.511 \mathrm{MeV}, 106 \mathrm{MeV}$ and $1777 \mathrm{Mev}$, respectively. The three species of neutrinos 
can inter-convert. This flip-flopping phenomena, which is referred to as 'neutrino oscillations', implies that the neutrinos have different masses. It is widely thought that neutrinos play decisive role in energy transport, since with their short-range, weak nuclear force they escape easily from all but the most dense plasmas. Furthermore, because neutrinos have mass, they thus play an important role in the formation of structures and ultimately in the fate of the universe and dense massive stars. Finally, in view of non-zero mass of wispy neutrinos, the standard theory of particle physics describing the fundamental constituents and their interactions in our cosmos should be re-examined.

It is well known that neutrinos interact with the background dense plasma through the weak nuclear force [1-6]. The dispersion relation for neutrino oscillations in plasmas is

$$
\left(E_{v}-V_{B}\right)^{2}-p_{v}^{2} c^{2}-m_{\mu} c^{2}=0,
$$

where $E_{v}=\hbar \omega_{v}$ is the neutrino energy, $\hbar\left(=1.0546 \times 10^{-27}\right.$ erg s) is the Planck's constant divided by $2 \pi, \omega_{v}$ is the neutrino oscillation frequency, $V_{B}=\sqrt{2} G n_{e}$ is the Bethe potential energy associated with the interaction of neutrinos with the plasma via the weak nuclear force, $G_{F}=9 \times 10^{-38} \mathrm{ev} \mathrm{cm}^{3}$ is the Fermi constant for weak nuclear interactions, $n_{e}$ is the electron number density, $\mathbf{p}_{v}=\hbar \mathbf{k}_{v}$ is the neutrino momentum, $\mathbf{k}_{v}$ is the propagation vector, $c$ is the speed of light in vacuum and $m_{\mu}$ is the neutrino mass. The neutrino energy density is $W_{v}=|\psi|^{2} / 4 \pi$. The evolution of the neutrino amplitude wave function, $\psi$, is governed by [5]

$$
\left(\hbar^{2} c^{2} \nabla^{2}-m_{\mu}^{2} c^{2}-\hbar^{2} \frac{\partial^{2}}{\partial t^{2}}\right) \psi \approx i 2 \sqrt{2} G n_{e} \hbar \frac{\partial \psi}{\partial t},
$$

which is obtained by letting $E=i \hbar \partial / \partial t$ and $\mathbf{p}_{v}=-i \hbar \nabla$ in (1) and operating the resultant equation on $\psi$.

In this paper we show that neutrino oscillations can be excited by a pre-existing electron density ripple in a dense plasma. We take the electron density as

$$
n_{e}=n_{0}[1+\epsilon \cos (k x)]
$$

where $\epsilon$ is the fractional perturbation ripple density, $k$ is the wave number of the ripple and $n_{0}$ is the equilibrium electron number density. The electron density ripple, which could be created by busts of intense photons [7], is assumed to be stationary because the timescale of the electron neutrino oscillations is much shorter than that for the background electron oscillations.

The one-dimensional propagation of neutrino oscillations is governed by the wave equation

$$
\left(\frac{\partial^{2}}{\partial x^{2}}-\frac{m_{v}^{2} c^{2}}{\hbar^{2}}-\frac{\omega_{v}^{2}}{c^{2}}\right) \varphi-\frac{2 \sqrt{2} G n_{e}}{\hbar c^{2}} \varphi=0,
$$

which is deduced from (2) by assuming that $\psi=\varphi \exp \left(-i \omega_{v} t\right)$. By using (3) in (4) we obtain

$$
\frac{\partial^{2} \varphi}{\partial x^{2}}+\frac{\Omega^{2}}{c^{2}}\left[1-\frac{K_{B}^{2} c^{2}}{\Omega^{2}} \epsilon \cos (k x)\right] \varphi=0,
$$

where $\Omega^{2}=\omega_{v}^{2}-\left(m_{\mu} c^{4} / \hbar^{2}\right)-K_{B}^{2} c^{2}$ and $K_{B}^{2}=2 \sqrt{2} G n_{0} / \hbar c^{2}$. 
Introducing $k x=2 \xi$ in (5), we can cast it in the form of the standard Mathieu equation $[8,9]$,

$$
\frac{\partial^{2} \varphi}{\partial \xi^{2}}+\frac{4 \Omega^{2}}{k^{2} c^{2}}[1-\beta \cos (2 \xi)] \varphi=0
$$

where $\beta=\epsilon K_{B}^{2} c^{2} / \Omega^{2}$. Equation (6) predicts instability for $\beta \ll 1$ and $[8,9]$

$$
\frac{2 \Omega}{k c}=p,
$$

where $p$ is an integer. Instability is strongest for $p \simeq 1$ and in that region the growth rate is

$$
\gamma=\frac{\Omega}{2}\left[\frac{\beta^{2}}{4}-(p-1)^{2}\right]^{1 / 2} .
$$

For larger $\beta$ one can have strong off-resonant growth rate. For $p \simeq 2$, 3 etc., the growth rates are smaller; the maximum growth rate for $\beta \ll 1$ is $\gamma / \Omega \simeq \beta^{2} / 8$ for $p=2$ and $\gamma / \Omega=81 \beta^{3} / 1024$ for $p=3[8]$.

To summarize, we have shown that a pre-existing electron density ripple can excite neutrino oscillations in dense plasmas. Physically, the energy stored in an electron density ripple is coupled to the electron neutrino oscillations by exciting neutrino oscillation sidebands due to the parametric interaction $[8,9]$. As a result, the electron neutrino oscillations with the angular frequency, $\omega_{v}=\left(\omega_{m}^{2}+K_{B}^{2} c^{2}+p^{2} k^{2} c^{2} / 4\right)^{1 / 2}$, would grow, where $\omega_{m}=m_{v} c^{2} / \hbar$. The neutrino oscillation frequency, $\omega_{v}$, is tunable by varying the electron density ripple wavelength, $2 \pi / k$. The growth rate is strongest for $p \simeq 1$. The non-thermal neutrino oscillations play a significant role in energy transport, as well as in the formation of structures and their dynamics in dense plasmas, such as those in supernovae and white dwarf stars. Furthermore, neutrinos with non-zero mass may have played a crucial role in the production of an excess of matter over anti-matter in our cosmos, and are thus intimately linked to our very existence.

\section{References}

[1] Bethe, H. A. 1986 Phys. Rev. Lett. 56, 1305.

[2] Bethe, H. A. 1990 Rev. Mod. Phys. 62, 801.

[3] Kuo, T. K. and Pantaleone, J. 1989 Rev. Mod. Phys. 62, 937.

[4] Bingham, R., Dawson, J. M., Su, J. J. and Bethe, H. A. 1994 Phys. Lett. A 193, 279.

[5] Bingham, R., Bethe, H. A., Dawson, J. M., Shukla, P. K. and Su, J. J. 1996 Phys. Lett. A 220, 107.

[6] Silva, L. O., Bingham, R., Dawson, J. M. et al., 1999 Phys. Rev. Lett. 83, 2703.

[7] Mendonça, J. T. 2001 Theory of Photon Acceleration. Bristol, UK: Institute of Physics.

[8] McLachlan, N. W. 1964 Theory and Application of Mathieu Equations, chapter IV. New York: Dover.

[9] Lin, A. T., Kaw, P. K. and Dawson, J. M. 1978 Phys. Rev. A 8, 2618. 